ARTICLE

Received 23 Jun 2014 | Accepted 10 Oct 2014 | Published 21 Nov $2014 \quad$ DOl: 10.1038/ncomms6527

\title{
Experimental protocol for high-fidelity heralded photon-to-atom quantum state transfer
}

\author{
Christoph Kurz ${ }^{1}$, Michael Schug ${ }^{1}$, Pascal Eich¹, Jan Huwer ${ }^{1}$, Philipp Müller $^{1}$ \& Jürgen Eschner ${ }^{1}$
}

A quantum network combines the benefits of quantum systems regarding secure information transmission and calculational speed-up by employing quantum coherence and entanglement to store, transmit and process information. A promising platform for implementing such a network are atom-based quantum memories and processors, interconnected by photonic quantum channels. A crucial building block in this scenario is the conversion of quantum states between single photons and single atoms through controlled emission and absorption. Here we present an experimental protocol for photon-to-atom quantum state conversion, whereby the polarization state of an absorbed photon is mapped onto the spin state of a single absorbing atom with $>95 \%$ fidelity, while successful conversion is heralded by a single emitted photon. Heralded high-fidelity conversion without affecting the converted state is a main experimental challenge, in order to make the transferred information reliably available for further operations. We record $>80 \mathrm{~s}^{-1}$ successful state transfer events out of $18,000 \mathrm{~s}^{-1}$ repetitions.

\footnotetext{
${ }^{1}$ Universität des Saarlandes, Experimentalphysik, Campus E2 6, 66123 Saarbrücken, Germany. Correspondence and requests for materials should be addressed to C.K. (email: c.kurz@physik.uni-saarland.de).
} 
T rapped single atomic ions are well suited for implementing quantum memories and processors, as they allow for long quantum information storage times and high-fidelity state manipulation and read-out ${ }^{1-4}$. Optical photons, on the other hand, are the most viable carriers of quantum information over long distances ${ }^{5,6}$. The integration of ion-based quantum processing nodes and photonic quantum communication in order to form a quantum network ${ }^{7,8}$ appears therefore particularly attractive. Remarkable fidelity and efficiency have been achieved in experiments on photon storage and read-out with atomic ensembles ${ }^{9}$; we focus on single-atom systems because of their prospect of processing the transmitted quantum information via local quantum gate operations ${ }^{10}$. Using single ions or atoms as senders and receivers of single photons requires controlling the emission ${ }^{11}$ and absorption processes, which, however, suffers from low efficiencies, most of all because of incomplete overlap between the spatial profile of the atomic emission or absorption and the photonic mode ${ }^{12}$. Even with approaches that employ optical resonators ${ }^{13-15}$ or a deep parabolic mirror ${ }^{16,17}$, results have so far remained limited to overall fidelities below $10 \%$. A possible remedy, which makes the fidelity of the state transfer process independent from its efficiency, is to filter out successful events by a heralding signal; this has been proposed ${ }^{18-20}$ and successfully applied for the creation of remote atom-atom entanglement ${ }^{21,22}$. It has also been proposed for mapping a photonic polarization state onto an atomic quantum bit by heralding the single-photon absorption with the detection of another single photon emitted in a Raman process ${ }^{23,24}$, but experimental approaches so far have only used a fluorescence signal as herald, which does not preserve the atomic state $e^{12,25,26}$.

Here we present the experimental implementation of a protocol for high-fidelity photon-to-atom state mapping heralded by Raman emission of a single photon. Out of $18,000 \mathrm{~s}^{-1}$ runs of the protocol, we obtain $>80 \mathrm{~s}^{-1}$ successful state transfer events with $97 \%$ state fidelity and $95 \%$ process fidelity. The single absorption events in this proof-of-principle experiment are driven by multi-photon laser pulses, but the results demonstrate that the state transfer protocol may be readily implemented with true single photons.

\section{Results}

Experimental protocol and setup. Figure 1 illustrates the principle of the experiment, which adapts an earlier proposal ${ }^{23}$ to our atomic system, a single ${ }^{40} \mathrm{Ca}^{+}$ion ${ }^{24}$. A superposition state in the $\mathrm{D}_{5 / 2}$ manifold is prepared as initial state for heralded absorption.
Photons at $854 \mathrm{~nm}$ wavelength excite this state to $\mathrm{P}_{3 / 2}$. For any photon polarization, the corresponding superposition of the $\sigma^{+}$ and $\sigma^{-}$transition is driven. Upon absorption of a photon, the ion returns to the $S_{1 / 2}$ ground state (with $93.5 \%$ probability, given by the $\mathrm{P}_{3 / 2}$ branching fraction ${ }^{27}$ ), releasing a single photon at $393 \mathrm{~nm}$ wavelength ${ }^{28}$. State mapping is completed by the detection of a $\pi$-polarized $393 \mathrm{~nm}$ photon; it heralds the absorption process while creating a superposition of the $S_{1 / 2}$ sublevels that corresponds to the polarization of the absorbed photon.

In order to verify the state transfer and quantify its fidelity, standard atomic-state analysis ${ }^{29}$ is performed after the detection of the heralding photon. For measuring the ion in the $| \pm 1 / 2\rangle$ basis of the $S_{1 / 2}$ manifold, the $|+1 / 2\rangle$ state is transferred (shelved) to the $\mathrm{D}_{5 / 2}$ manifold. Switching on the cooling lasers then either reveals a fluorescence signal (ion in $|-1 / 2\rangle$ ) or not (ion in $|+1 / 2\rangle$ ). For projecting onto a superposition basis of $| \pm 1 / 2\rangle$, a resonant radio-frequency (RF) pulse effects a basis rotation before shelving.

Our experimental setup is sketched in Fig. 2. A single ${ }^{40} \mathrm{Ca}^{+}$ion is trapped in a linear radio-frequency (Paul) trap and Doppler cooled by frequency-stabilized diode lasers ${ }^{30}$. Cooling is facilitated by a static magnetic field, also defining the quantization axis. A narrow-band laser at $729 \mathrm{~nm}$ is used for coherent manipulations on the $\mathrm{S}_{1 / 2}-\mathrm{D}_{5 / 2}$ transition. A laser at $854 \mathrm{~nm}$ with variable polarization provides the photons to excite the $\mathrm{D}_{5 / 2}-\mathrm{P}_{3 / 2}$ transition. For the purpose of demonstrating our experimental protocol, we send many identical photons to the atom in each individual run; quantum state transfer from a single photon to the atom would be attained by the same procedure. In order to coherently control the magnetic-dipole transition coupling the two $S_{1 / 2}$ Zeeman states, we use a copper-wire coil placed below the trap. An RF current at $7.8 \mathrm{MHz}$ creates an oscillating magnetic field (perpendicular to the quantization axis) at the position of the ion, driving the magnetic-dipole transition. Blue photons emitted by the ion are collected through two in-vacuum high-numerical aperture (NA) laser objectives $\left(\right.$ HALOs) ${ }^{31}$. Photons at $393 \mathrm{~nm}$ wavelength are filtered by a polarizer such that only those scattered on $\pi$ transitions are transmitted. The photons are then coupled to optical fibers and detected by photomultiplier tubes whose output pulses are fed into the sequence-control unit. For single photons at $393 \mathrm{~nm}$, the pulses are in addition time-tagged and stored for later processing.

State transfer in circular polarization basis. In a first experiment, we choose the $854 \mathrm{~nm}$ light polarization to be right-handed

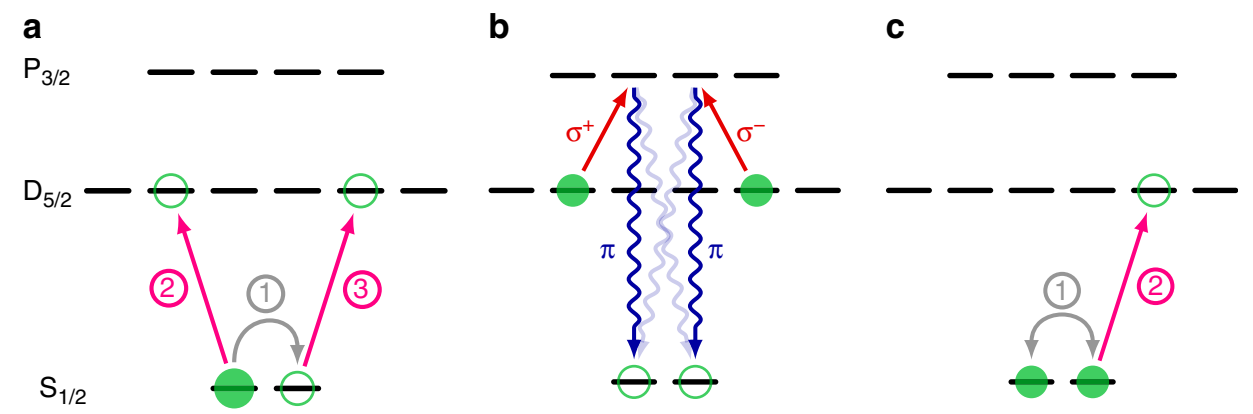

Figure 1 | Experimental scheme for photon-polarization storage. Black bars denote the Zeeman sublevels of the involved atomic levels $S_{1 / 2}$, $D_{5 / 2}$ and $P_{3 / 2}$. (a) Preparation: first the ion is optically pumped into the $|-1 / 2\rangle$ Zeeman sublevel of the $S_{1 / 2}$ ground-state manifold (filled green circle); then a sequence of one radio-frequency (RF, gray arrow) and two optical (pink arrows) pulses creates a coherent superposition between $\left|D_{5 / 2}, m=-3 / 2\right\rangle$ and $\left|D_{5 / 2}, m=+3 / 2\right\rangle$ (open green circles). (b) Storage: the absorption of an $854 \mathrm{~nm}$ photon of arbitrary polarization (red arrows) triggers the emission of a single $393 \mathrm{~nm}$ photon (wavy arrows). The detection of a $\pi$-polarized $393 \mathrm{~nm}$ photon (dark blue) projects the atom into a superposition state in $\mathrm{S}_{1 / 2}$ that corresponds to the polarization of the absorbed $854 \mathrm{~nm}$ photon. (c) Read-out: electron shelving (pink arrow) and atomic-fluorescence detection distinguish the $\mathrm{S}_{1 / 2}$ sublevels; depending on the desired measurement basis, another RF pulse (gray arrow) is applied before. 


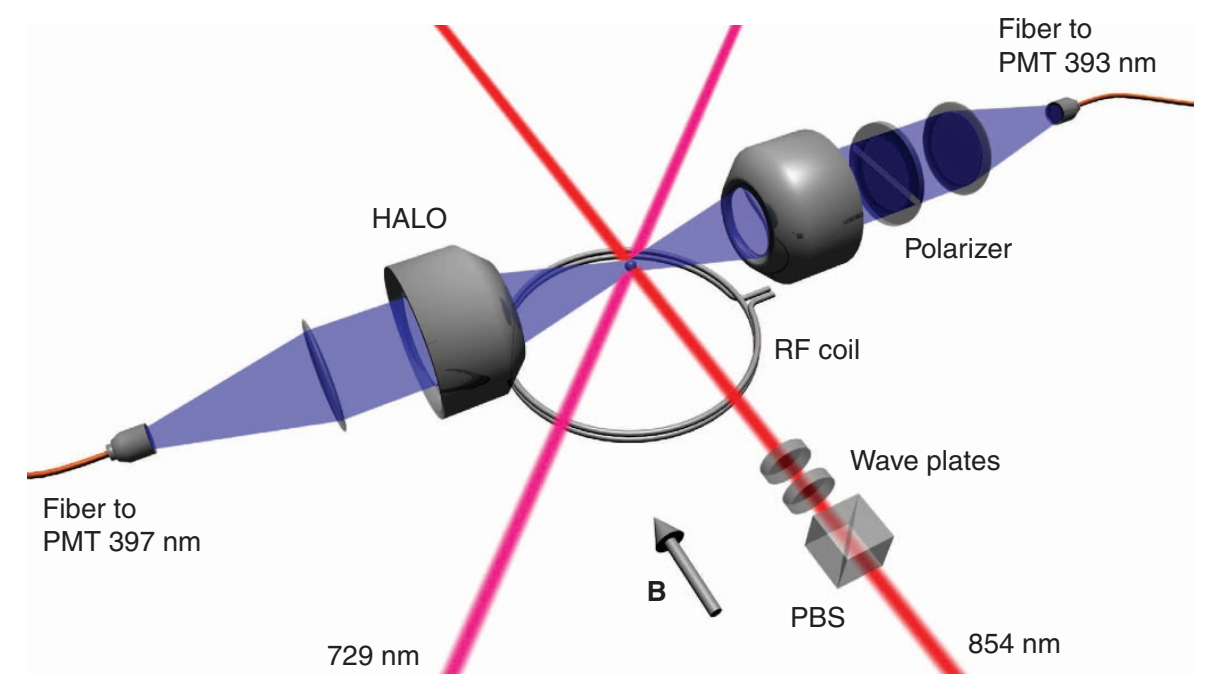

Figure 2 | Experimental setup. HALO, high-NA laser objective; PMT, photomultiplier tube; PBS, polarizing beam splitter; B, magnetic-field direction. The ion is trapped between the HALOs.

circular, driving $\sigma^{+}$transitions in the atomic system. Hence, only the population in the $\left|D_{5 / 2}, m=-3 / 2\right\rangle$ state is excited. The detection of a single $393 \mathrm{~nm}$ photon in the state $|\pi\rangle$ then ideally leaves the ion in the state $|-1 / 2\rangle$. After detecting a photon, the atomic state is measured in the $| \pm 1 / 2\rangle$ basis. According to the measurement outcome, the photon arrival times are sorted into two histograms. As can be seen from Fig. 3a, the emission of photons followed by a projection of the atomic state onto $|+1 / 2\rangle$ is almost completely suppressed. As a reasonable trade-off between high quantum-state fidelity and high photondetection efficiency, we select only detection events within the first $450 \mathrm{~ns}$ of the photonic wave packet. From the number of events in each histogram within this time window, we obtain a fidelity with respect to the ideal atomic state $|-1 / 2\rangle$ of $97.8(1) \%$. The measurement was then repeated for left-handed circularly polarized $854 \mathrm{~nm}$ light, yielding a fidelity of the atomic state with respect to $|+1 / 2\rangle$ of $96.4(3) \%$.

State transfer of linear polarization. In a second step, we change the $854 \mathrm{~nm}$ light polarization to linear. Thus, a superposition of both $\sigma$ transitions is driven, with the relative phase given by the angle of the linear polarization (which is defined with respect to the plane spanned by the axis of fluorescence collection and the quantization axis). The detection of a scattered $|\pi\rangle$ photon then heralds the projection of the atomic state onto the superposition of $|+1 / 2\rangle$ and $|-1 / 2\rangle$ that corresponds to the $854 \mathrm{~nm}$ light polarization. Finally, a $\pi / 2 \mathrm{RF}$ pulse is applied before shelving and fluorescence detection, thereby measuring the atomic state in the superposition basis. Figure $3 \mathrm{~b}$ shows the arrival-time histograms for the two possible measurement outcomes. The oscillations visible in the shapes of the conditional photon wave packets are explained by the difference between the Larmor frequency of the initial superposition of $\left|D_{5 / 2}, m= \pm 3 / 2\right\rangle$ and that of the final superposition of $\left|S_{1 / 2}, m= \pm 1 / 2\right\rangle$. Taking into account the Landé factors, the oscillation period is given by $T=h /\left(1.6 \mu_{\mathrm{B}} B\right)=160 \mathrm{~ns}$ for a static magnetic field $B=2.8 \mathrm{G}$. In addition, the wave packets are approximately twice as long as for circularly polarized $854 \mathrm{~nm}$ light, as linear polarizations have a relative overlap with $\sigma$ transitions of only $\frac{1}{\sqrt{2}}$. This results in lower Rabi frequencies and hence longer wave packets.

In order to show that the detection of a Raman-scattered photon indeed heralds the successful transfer of the polarization
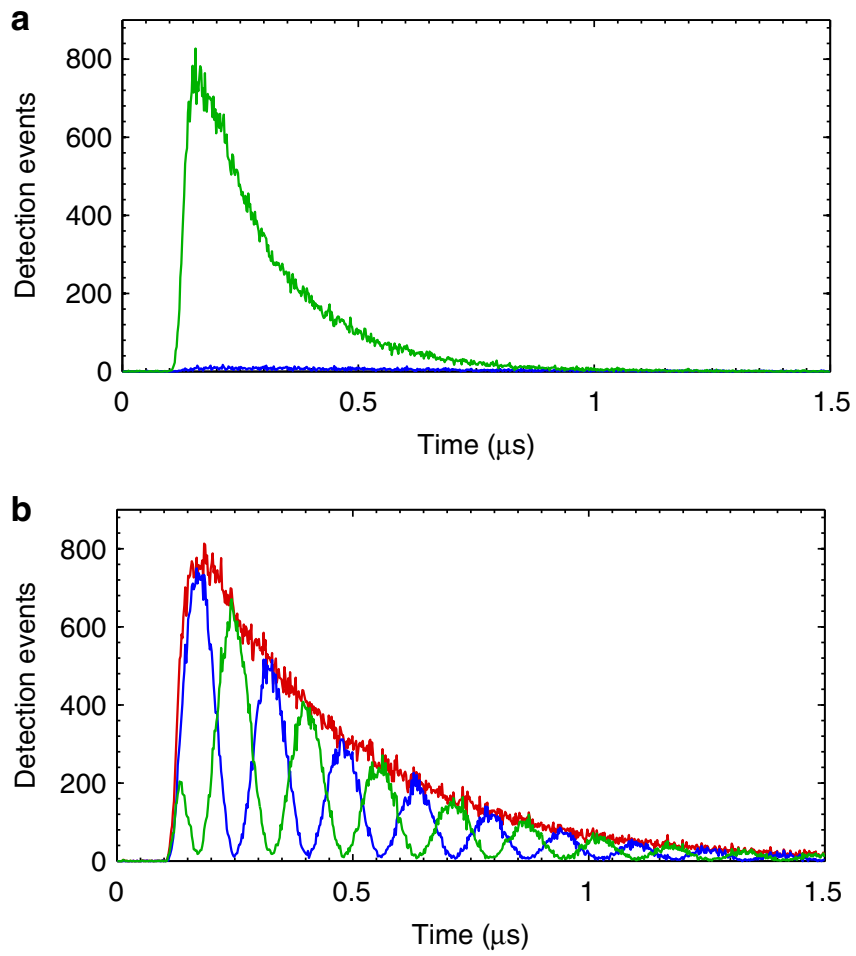

Figure 3 | Conditional arrival-time histograms of single $393 \mathrm{~nm}$ photons. (a) Projection onto $|+1 / 2\rangle$ (blue) and $|-1 / 2\rangle$ (green) for right-handed circularly polarized $854 \mathrm{~nm}$ light. (b) Projection onto the superposition basis for linearly polarized $854 \mathrm{~nm}$ light. The red curve shows the sum of the two histograms, that is, the unconditional arrival-time distribution. For each graph, the bin size is $2 \mathrm{~ns}$ and the overall measuring time is $20 \mathrm{~min}$.

state of a laser photon onto the atomic ground state, we analyze contrast and phase of the oscillations in the Raman-photon wave packets. As the phase accumulated in the $\left|D_{5 / 2}, m= \pm 3 / 2\right\rangle$ superposition before returning to the ground state is directly given by the time duration until the photon is detected, each detection-time value $t$ converts into a phase value $\varphi=2 \pi \frac{t}{T}$. As detection times differing by integer multiples of the Larmor period $T$ yield equivalent final atomic states, we use the reduced 


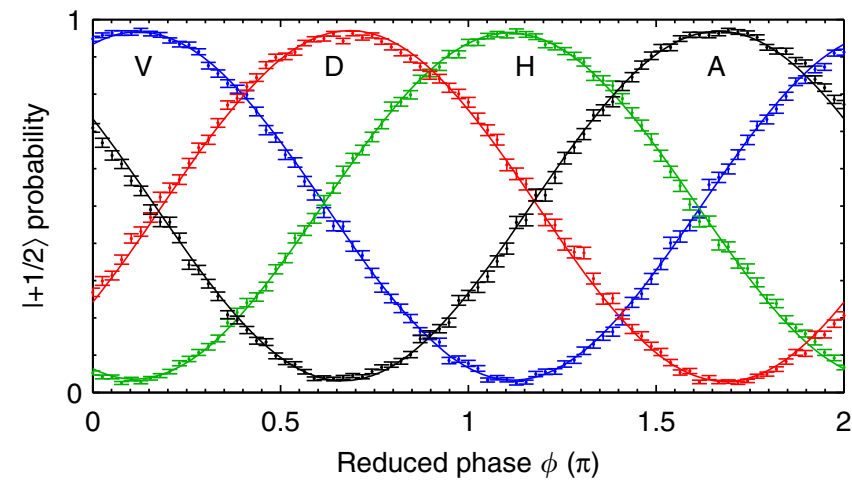

Figure 4 | Atomic-state analysis. Shown is the probability to find the ion in state $|+1 / 2\rangle$ after the $\pi / 2 \mathrm{RF}$ pulse as a function of the reduced phase $\phi$ for the $854 \mathrm{~nm}$ light polarizations vertical $(V)$, horizontal $(H)$, diagonal (D) and antidiagonal (A). The solid lines are sinusoidal fits.

phase $\phi=\varphi \bmod 2 \pi$. For each such phase, we derive from the arrival-time histograms the probability of projecting the ion onto the state $|+1 / 2\rangle$ after the $\pi / 2 \mathrm{RF}$ pulse. For the $450 \mathrm{~ns}$ time window, these probabilities are depicted in Fig. 4 for different linear polarizations of the $854 \mathrm{~nm}$ light. We observe the expected sinusoidal behaviour as a function of the reduced phase $\phi$, as well as the expected consecutive relative phase shift of $\pi / 2$ going from $\mathrm{V}$ to $\mathrm{D}, \mathrm{H}$ and $\mathrm{A}$ polarized light. From the fitted visibility values $V$, we obtain the fidelities $F$ of the four generated atomic states with respect to the ideal states corresponding to $\mathrm{V}, \mathrm{H}, \mathrm{D}$ and $\mathrm{A}$ polarization, $F=\frac{1}{2}(1+V)=96.7(1) \%, 96.4(1) \%, 97.0(2) \%$ and $96.9(2) \%$, respectively. Including the previously determined fidelities for circular polarization, we arrive at an average fidelity of all six reconstructed atomic states with respect to the ideal states of $96.9(1) \%$.

The observed quantum-state fidelities are mainly limited by two effects: first, detector dark counts mimic the detection of $393 \mathrm{~nm}$ photons (1.7\%). Second, spontaneous decay of population from the $\mathrm{P}_{3 / 2}$ back to the $\mathrm{D}_{5 / 2}$ state leaves the ion in a mixed state from which it is subsequently transferred to $S_{1 / 2}$; the observed contribution (1.4\%) is on the order of what is expected from the $\mathrm{P}_{3 / 2}$ branching fractions. This limitation will not matter in experiments employing true single photons at $854 \mathrm{~nm}$ as there is no second photon available for re-exciting the ion. The state fidelity may also be reduced by magnetic-field noise, but here the other two effects were more relevant; in a different realization of the protocol, described below, magnetic-field noise was found to be significant.

Quantum process tomography. Another way of quantifying the transfer fidelity of the mapping process is to perform quantum process tomography. The quantum process $\varepsilon$ mapping the photon polarization state $\rho$ onto the atomic state can be expressed as $\varepsilon(\rho)=\sum_{m, n} \chi_{m n} \sigma_{m} \rho \sigma_{n}$ with the Pauli matrices $\left\{\sigma_{i=1, \ldots 4}\right\}=$ $\left\{1, \sigma_{x}, \sigma_{y}, \sigma_{z}\right\}$ and the process matrix $\chi$ (ref. 32). The value $\left|\chi_{11}\right|$ represents the identity part of the quantum process and is known as the process fidelity. It can be computed using four mutually unbiased polarization states and the resulting atomic states. For a $450 \mathrm{~ns}$ time window, we derive a process fidelity of $95.0(2) \%$, with which the atomic state reproduces the polarization of the absorbed laser photon. It is instructive to analyze the figures of merit of the transfer for different choices of the time window. Figure 5 shows the process fidelity and average state fidelity as a function of the photon-detection probability, the latter being set by the choice of the detection-time window. For our $450 \mathrm{~ns}$ time window, we achieve $0.438(1) \%$ average detection efficiency.

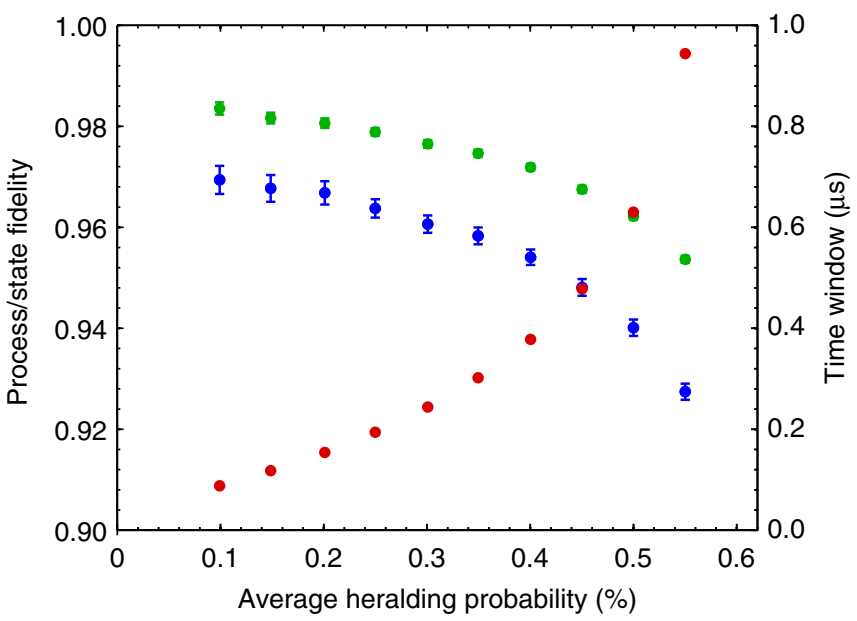

Figure 5 | State-transfer fidelity. Process fidelity (blue dots) and average quantum-state fidelity (green dots), characterizing the mapping process, for different heralding probabilities (set through the time-window length indicated by red dots).

\section{Discussion}

Reference 24 discusses various schemes for mapping the polarization state of an absorbed $854 \mathrm{~nm}$ photon onto the atomic ground-state qubit, of which we also implemented an alternative one: instead of preparing the ion in a coherent superposition of the $\left|D_{5 / 2}, m= \pm 3 / 2\right\rangle$ states for absorption, we create a superposition of the states $\left|D_{5 / 2}, m= \pm 5 / 2\right\rangle$. The $854 \mathrm{~nm}$ light then transfers the ion to the $S_{1 / 2}$ ground state by emitting a $393 \mathrm{~nm}$ photon on either the $\sigma^{+}$or $\sigma^{-}$transition. For linearly polarized $854 \mathrm{~nm}$ light, the polarization of the scattered photon is maximally entangled with the final atomic spin state: $\left|\Psi_{\text {ion }- \text { photon }}\right\rangle=\frac{1}{\sqrt{2}}\left(\left|+\frac{1}{2}\right\rangle\left|\sigma^{+}\right\rangle+\mathrm{e}^{i \theta}\left|-\frac{1}{2}\right\rangle\left|\sigma^{-}\right\rangle\right)$. As emitted photons are collected perpendicularly to the quantization axis, only one specific superposition of $\left|\sigma^{+}\right\rangle$and $\left|\sigma^{-}\right\rangle$is observed (after removing the polarizer). The detection of the scattered photon thus projects the atomic state onto the superposition of $|+1 / 2\rangle$ and $|-1 / 2\rangle$ that corresponds to the $854 \mathrm{~nm}$ light polarization. For the $450 \mathrm{~ns}$ detection-time window, we achieve $91.7(5) \%$ process fidelity, $94.8(2) \%$ average state fidelity and $0.318(1) \%$ heralding probability. The reduced fidelity as compared with the previous scheme is mostly attributed to the non-linearly ${ }^{33}$ higher sensitivity of the $| \pm 5 / 2\rangle$ states to magnetic-field noise.

In conclusion, we demonstrated heralded, high-fidelity mapping of a photonic polarization state onto an atomic quantum bit. After preparing a single ${ }^{40} \mathrm{Ca}^{+}$ion in a coherent superposition state, the absorption of a photon on the $\mathrm{D}_{5 / 2}-\mathrm{P}_{3 / 2}$ transition maps the photonic polarization state onto the atomic $S_{1 / 2}$ Zeeman qubit. Successful mapping is heralded by the detection of a Raman-scattered photon at $393 \mathrm{~nm}$ wavelength, without perturbing the atomic qubit state. By virtue of our high-NA light-collection optics, the heralding probability amounts to $0.438(1) \%$. The process fidelity is $95.0(2) \%$ and the average fidelity of the resulting atomic state with respect to the initial polarization amounts to $96.9(1) \%$. Although in this demonstration we used laser photons to obtain a high interaction rate, the scheme can readily be applied to single photons, for example, from a spontaneous parametric down-conversion (SPDC) source ${ }^{34}$. Upon absorption of one photon, detection of a $393 \mathrm{~nm}$ Raman photon will then herald the entanglement of the partner photon with the ion. This scheme shall be extended to having both entangled partner photons absorbed by ions in remote traps ${ }^{35}$. Coincidental detection of two $393 \mathrm{~nm}$ photons 
then heralds the entanglement of the two spatially separated ions and completes photon-to-atom entanglement transfer.

\section{Methods}

Experimental sequence. All laser beams are controlled through acousto-optic modulators. The sequence starts with a $5 \mu$ s pump pulse of $\sigma^{-}$-polarized light at $397 \mathrm{~nm}$. This prepares the ion in the $|-1 / 2\rangle$ state of the $S_{1 / 2}$ manifold with 99.82(1)\% probability. Then a coherent superposition of the $| \pm 1 / 2\rangle$ states is created by an RF pulse of $2.8 \mu$ s duration. Subsequent $729 \mathrm{~nm}$ pulses, each lasting $9.6 \mu \mathrm{s}$, transfer the superposition to the $| \pm 3 / 2\rangle$ states of the $D_{5 / 2}$ manifold. The $854 \mathrm{~nm}$ beam from which a single photon is absorbed is switched on for $3 \mu$ s at $12 \mu \mathrm{W}$ optical power, yielding $\sim 9 \mathrm{MHz}$ Rabi frequency for a $125 \mu \mathrm{m}$ beam waist. If the Raman-scattered photon is not detected (in most cases), we apply Doppler cooling for $10 \mu \mathrm{s}$. Including delays in the control electronics, we achieve a repetition rate of $18 \mathrm{kHz}$.

Fluorescence detection. Light emitted by the ion is collected through two highNA laser objectives $(\mathrm{NA}=0.4$ ), each covering $\sim 4.2 \%$ of the total solid angle, and coupled to multi-mode optical fibers with $83 \%$ efficiency. The light is then detected by photomultiplier tubes with $\sim 28 \%$ quantum efficiency. For isotropic emission, this yields a total detection efficiency of $0.98 \%$.

Atomic-state analysis. After detecting the Raman photon at $393 \mathrm{~nm}$, the atomic state is analyzed: depending on whether a superposition basis is used, first a rotation in the $S_{1 / 2}$ manifold is performed by another RF pulse. Then we apply a shelving pulse at $729 \mathrm{~nm}$, which transfers the $|+1 / 2\rangle$ state to the $D_{5 / 2}$ manifold, before switching on the cooling lasers. A projection onto the $S_{1 / 2}$ state is signaled by the onset of resonance fluorescence, detected at a rate of $1.07 \times 10^{5} \mathrm{~s}^{-1}$. A projection onto $D_{5 / 2}$ leaves the ion dark, resulting in a detection rate of $60 \mathrm{~s}^{-1}$ from detector dark counts and laser stray light. Integrating the detection rate for $100 \mu \mathrm{s}$ allows us to discriminate between the two atomic states with $99.986 \%$ fidelity.

Indistinguishability of $\mathbf{3 9 3} \mathbf{~ n m}$ photons. It is important to note that in a singlephoton realization of the protocol, the arrival-time distribution of the Ramanscattered photon at $393 \mathrm{~nm}$ will not contain any information about the polarization of the single absorbed $854 \mathrm{~nm}$ photon. With $854 \mathrm{~nm}$ laser light we observe different distributions for different polarizations, as in Fig. 3a,b. For a single-photon realization, however, the relevant quantity is the peak value of this measured distribution, which is identical for the two cases; the subsequent shape only reflects the temporal behavior of the $\mathrm{D}_{5 / 2}$-state population for the case of laser excitation.

\section{References}

1. Leibfried, D. et al. Experimental demonstration of a robust, high-fidelity geometric two ion-qubit phase gate. Nature 422, 412-415 (2003).

2. Langer, C. et al. Long-lived qubit memory using atomic ions. Phys. Rev. Lett. 95, 060502 (2005).

3. Benhelm, J., Kirchmair, G., Roos, C. F. \& Blatt, R. Towards fault-tolerant quantum computing with trapped ions. Nat. Phys 4, 463-466 (2008).

4. Schindler, P. et al. A quantum information processor with trapped ions. New J. Phys. 15, 123012 (2013).

5. Weihs, G., Jennewein, T., Simon, C., Weinfurter, H. \& Zeilinger, A. Violation of Bell's inequality under strict Einstein locality conditions. Phys. Rev. Lett. 81, 5039 (1998).

6. Ma, X.-S. et al. Quantum teleportation over 143 kilometres using active feedforward. Nature 489, 269-273 (2012).

7. Kimble, H. J. The quantum internet. Nature 453, 1023-1030 (2008).

8. Duan, L.-M. \& Monroe, C. Quantum networks with trapped ions. Rev. Mod. Phys. 82, 1209 (2010).

9. Tanji, H., Ghosh, S., Simon, J., Bloom, B. \& Vuletić, V. Heralded single-magnon quantum memory for photon polarization states. Phys. Rev. Lett. 103, 043601 (2009).

10. Hucul, D. et al. Modular entanglement of atomic qubits using both photons and phonons. Preprint at http://arxiv.org/abs/1403.3696 (2014).

11. Stute, A. et al. Quantum-state transfer from an ion to a photon. Nat. Photon. 7, 219-222 (2013).

12. Schug, M., Huwer, J., Kurz, C., Müller, P. \& Eschner, J. Heralded photonic interaction between distant single ions. Phys. Rev. Lett. 110, 213603 (2013).

13. Boozer, A. D., Boca, A., Miller, R., Northup, T. E. \& Kimble, H. J. Reversible state transfer between light and a single trapped atom. Phys. Rev. Lett. 98, 193601 (2007).
14. Specht, H. P. et al. A single-atom quantum memory. Nature 473, 190-193 (2011).

15. Ritter, S. et al. An elementary quantum network of single atoms in optical cavities. Nature 484, 195-200 (2012).

16. Maiwald, R. et al. Stylus ion trap for enhanced access and sensing. Nat. Phys. 5, 551-554 (2009).

17. Fischer, M. et al. Efficient saturation of an ion in free space. Appl. Phys. B. (in press) doi:10.1007/s00340-014-5817-y (2014).

18. Cabrillo, C., Cirac, J. I., García-Fernández, P. \& Zoller, P. Creation of entangled states of distant atoms by interference. Phys. Rev. A 59, 1025 (1999).

19. Feng, X.-L., Zhang, Z.-M., Li, X.-D., Gong, S.-Q. \& Xu, Z.-Z. Entangling distant atoms by interference of polarized photons. Phys. Rev. Lett. 90, 217902 (2003).

20. Simon, C. \& Irvine, W. T. M. Robust long-distance entanglement and a loophole-free Bell test with ions and photons. Phys. Rev. Lett. 91, 110405 (2003).

21. Moehring, D. L. et al. Entanglement of single-atom quantum bits at a distance. Nature 449, 68-71 (2007).

22. Hofmann, J. et al. Heralded entanglement between widely separated atoms. Science 337, 72-75 (2012).

23. Lloyd, S., Shahriar, M. S., Shapiro, J. H. \& Hemmer, P. R. Long distance, unconditional teleportation of atomic states via complete Bell state measurements. Phys. Rev. Lett. 87, 167903 (2001).

24. Müller, P. \& Eschner, J. Single calcium-40 ion as quantum memory for photon polarization: a case study. Appl. Phys. B 114, 303-306 (2014).

25. Piro, N. et al. Heralded single-photon absorption by a single atom. Nat. Phys. 7, 17-20 (2011).

26. Huwer, J. et al. Photon entanglement detection by a single atom. New J. Phys. 15, 025033 (2013).

27. Gerritsma, R. et al. Precision measurement of the branching fractions of the $4 \mathrm{p}^{2} \mathrm{P}_{3 / 2}$ decay of Ca II. Eur. Phys. J. D 50, 13-19 (2008).

28. Kurz, C., Huwer, J., Schug, M., Müller, P. \& Eschner, J. A high-rate source for single photons in a pure quantum state. New J. Phys. 15, 055005 (2013).

29. Häffner, H., Roos, C. F. \& Blatt, R. Quantum computing with trapped ions. Phys. Rep. 469, 155-203 (2008).

30. Rohde, F. et al. A diode laser stabilization scheme for ${ }^{40} \mathrm{Ca}^{+}$single-ion spectroscopy. J. Phys. B 43, 115401 (2010).

31. Gerber, S. et al. Quantum interference from remotely trapped ions. New J. Phys. 11, 013032 (2009).

32. Chuang, I. L. \& Nielsen, M. A. Prescription for experimental determination of the dynamics of a quantum black box. J. Mod. Opt. 44, 2455-2467 (1997).

33. Mount, E. et al. Single qubit manipulation in a microfabricated surface electrode ion trap. New J. Phys. 15, 093018 (2013).

34. Haase, A., Piro, N., Eschner, J. \& Mitchell, M. W. Tunable narrowband entangled photon pair source for resonant single-photon single-atom interaction. Opt. Lett. 34, 55-57 (2009).

35. Sangouard, N., Bancal, J.-D., Müller, P., Ghosh, J. \& Eschner, J. Heralded mapping of photonic entanglement into single atoms in free space: proposal for a loophole-free Bell test. New J. Phys. 15, 085004 (2013).

\section{Acknowledgements}

We acknowledge support by the BMBF (QuOReP project, QSCALE Chist-ERA project) and the German Scholars Organization/Alfried Krupp von Bohlen und Halbach-Stiftung.

\section{Author contributions}

C.K., M.S. and P.E. contributed equally to this work; J.H. set up the narrow-band laser; C.K., M.S. and P.E. prepared the experiment and acquired and analyzed the data; P.M. performed numerical calculations; J.E. planned and supervised the project; C.K. and J.E. wrote the manuscript.

\section{Additional information}

Competing financial interests: The authors declare no competing financial interest.

Reprints and permission information is available online at http://npg.nature.com/ reprintsandpermissions/

How to cite this article: Kurz, C. et al. Experimental protocol for high-fidelity heralded photon-to-atom quantum state transfer. Nat. Commun. 5:5527 doi: $10.1038 /$ ncomms6527 (2014) 